\title{
Energy Harvesting in Delay-Aware Cognitive Shared Access Networks
}

\author{
Zheng Chen, Nikolaos Pappas and Marios Kountouris \\ Conference Publication
}

\section{Tweet}

N.B.: When citing this work, cite the original article.

Original Publication:

Zheng Chen, Nikolaos Pappas and Marios Kountouris, Energy Harvesting in Delay-Aware Cognitive Shared Access Networks, IEEE Workshop on Emerging Energy Harvesting Solutions for 5G Networks (5G-NRG), 2017

Copyright: www.ieee.org

Postprint available at: Linköping University Electronic Press

http://urn.kb.se/resolve?urn=urn:nbn:se:liu:diva-135319

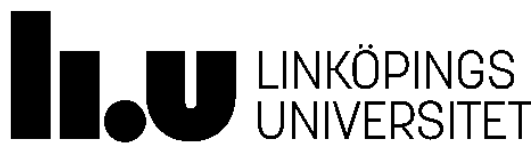




\title{
Energy Harvesting in Delay-Aware Cognitive Shared Access Networks
}

\author{
Zheng Chen*, Nikolaos Pappas ${ }^{\dagger}$, Marios Kountouris ${ }^{\ddagger}$ \\ * Department of Electrical Engineering, Linköping University, Linköping, Sweden \\ ${ }^{\dagger}$ Department of Science and Technology, Linköping University, Campus Norrköping, Sweden \\ $¥$ Mathematical and Algorithmic Sciences Lab, France Research Center, Huawei Technologies France SASU, \\ Boulogne-Billancourt, France \\ Email: zheng.chen@liu.se, nikolaos.pappas@liu.se,marios.kountouris@huawei.com
}

\begin{abstract}
In this work, we study the effect of energy harvesting in a cognitive shared access network with delay constraints on the primary user. We model the distribution of secondary nodes by a homogeneous Poisson point process (PPP), while the primary user is located at fixed location. The secondary users are assumed to have always packets to transmit whilst the primary transmitter has bursty traffic. We assume an energy harvesting zone around the primary transmitter and a guard zone around the primary receiver. The secondary users are transmitting in a random access manner, however, transmissions of secondary nodes are restricted by their battery status and location. Targeting at achieving the maximum secondary throughput under primary delay constraints, we analyze the impact of various parameters on the performance of the considered network. Our results provide insights into the optimization of access protocol parameters for the energy harvesting-based cognitive shared access network with delay constraints.
\end{abstract}

\section{INTRODUCTION}

Radio-frequency (RF) energy harvesting has been investigated extensively in recent years for its ability to turn ambient energy into power source for electronic devices. Enabling wireless charging on low-power devices shows many advantages and brings new opportunities in the design of future wireless networks. In an environment where battery replacement is expensive or not feasible, user devices with energy harvesting capabilities can opportunistically access wireless networks whenever sufficient energy is harvested.

In a typical cognitive radio network, a secondary user can access the wireless channel to transmit a packet only when the primary user is idle. By allowing the secondary user to harvest energy when the primary node is transmitting, the energy efficiency of such system can be largely improved [1][3]. In collision-based cognitive radio, the optimal spectrum sensing policy that maximizes the aggregate throughput with an energy causality constraint has been studied in [1], [2]. Under a similar setup, with the assumption that the secondary user can infer knowledge from the channel with an online learning algorithm, the optimal channel access policy has been studied in [3]. Note that all the aforementioned studies

This work was supported in part by the Swedish Foundation for Strategic Research (SSF), the Swedish Research Council (VR), ELLIIT, and the European Unions Seventh Framework Programme (FP7/2007-2013) under grant agreement no 324515 (MESH-WISE). assume one single secondary link and a collision channel model. The work in [4] considers a cognitive network with multi-packet reception (MPR) capabilities at both primary and secondary nodes. When the primary user is transmitting, the secondary user can access the spectrum/channel with a random probability that is optimized to maximize the stable throughput.

Spatial point processes have been widely used in wireless networks to model the node distribution in large topologies. Prior studies in the application of stochastic geometry in wireless networks provide tractable analytical results on the network performance metrics based on the signal-to-interferenceplus-noise (SINR) distribution in random networks with spatially distributed nodes [5]. With the help of stochastic geometry, energy harvesting in random wireless networks has been studied in many different cases in the literature. The spatial throughput of mobile ad hoc networks with energy harvesting nodes is investigated in [6]. The effect of energy harvesting in geometry-based cognitive radio networks has been studied in [7] and [8], where a secondary node can access the spectrum when it harvests enough RF energy and when it is outside the guard zone of the primary user. Another potential application of energy harvesting is in device-to-device (D2D) underlaid cellular networks where D2D-enabled user devices can harvest energy from cellular transmission. The performance analysis and optimization in such networks have been studied in [9], [10]. Note that in these works the secondary users can opportunistically access the spectrum when certain criteria are satisfied. Furthermore, the primary nodes are often assumed to be constantly transmitting packets to their destinations. The effect of random packet arrivals at the primary nodes on the network performance has been overlooked in cognitive shared access networks with energy harvesting-based opportunistic secondary users. This motivates our study to consider the average packet delay of the primary user, which contains also the queueing delay, when optimizing the performance of such networks. Additionally, there are some works that consider queueing analysis in energy harvesting networks and study the effect of energy harvesting on the stability region of small networks [11]-[13].

In this work, we consider a cognitive shared access network 
which consists of one primary pair and many randomly distributed secondary pairs. A secondary transmitter can opportunistically access the primary spectrum with a certain probability when: 1) it is fully charged; 2) it is outside the guard zone around the primary receiver. Inspired by the network model in [14], [15], we take into account the queueing delay and the transmission delay of the primary user while assuming bursty packet arrivals at the primary transmitter. The interaction between the primary and the secondary transmission allows us to optimize the primary guard zone and secondary access probability in order to achieve the maximum secondary throughput under the primary delay constraint.

\section{SYSTEM MODEL}

We consider the following cognitive shared access network model. The primary transmitter (PT) is located at the center of the network region modeled by a disk $\mathcal{C}$ of radius $R$, while the primary receiver (PR) is located at fixed location $y_{0}$ with distance $d_{\mathrm{p}}$ to the PT. The secondary transmitters (STs) are distributed according to a homogeneous Poisson Point Process (PPP) $\Phi_{s}$ with intensity $\lambda_{s}$. Their associated receivers are located at isotropic directions with fixed link distance $d_{\mathrm{s}}$. We assume that each ST is equipped with a radio frequency (RF) energy harvester that can turn the received ambient RF energy into Direct Current (DC) power. In the literature of RF energy harvesting, one common assumption is the energy harvesting $(E H)$ zone, which defines the region within which the RF energy can be harvested [8], [9]. This assumption comes from the fact that a threshold power level must be reached in order to trigger the power conversion. We define the energy harvesting zone by a disk with radius $r_{\mathrm{eh}}$ centered at the primary transmitter (PT). When a ST falls in the energy harvesting zone of the PT, the ST will be fully charged within one time slot, while the charged battery can support one-slot transmission of the ST [6], [8]. As the result, in each network configuration, the STs that can be charged by the PT transmission are denoted by the set $\Phi_{s} \cap \mathcal{B}\left(0, r_{\mathrm{eh}}\right)$, where $\mathcal{B}(a, b)$ denotes the disk centered at $a$ with radius $b$. We denote the primary and secondary transmission power by $P_{1}$ and $P_{2}$, respectively, with $P_{2} \ll P_{1}$.

Due to the spectrum sharing between the primary and secondary nodes, the aggregate secondary interference received at the PR might largely degrade the performance of the primary user. In order to prevent the STs from being very close to the $\mathrm{PR}$, we impose a guard zone around the PR with radius $r_{\mathrm{gz}}$ where no active ST can lie in [16]. Therefore, a ST is allowed to transmit in a time slot when

- it has harvested enough RF energy, i.e., it was within the primary EH zone;

- it is outside the primary guard zone in the current time slot.

To exploit potential throughput improvement, one common and simple strategy for interference management is the random access, also referred to as spatial ALOHA, which means that each node accesses the spectrum/channel with a certain probability, independently of other nodes in the network [17].
It is shown that by properly assigning the access probability, denoted by $p_{\mathrm{s}}$, the network throughput can be largely improved compared to the case that all the nodes are active.

In order to clarify the notations, we define the following probabilities:

- $p_{\text {ch }}$ denotes the probability that a typical ST is fully charged at the beginning of a time slot;

- $p_{\text {tr }}$ denotes the probability that a typical ST will be transmitting in this time slot;

- $p_{\mathrm{gz}}$ denotes the probability that a typical ST is not within the primary guard zone;

- $p_{\text {eh }}$ denotes the probability that a typical ST lies in the EH zone of the PT.

Here, the typical ST refers to a reference point in the network region based on which we perform statistical analysis of the network performance. From Slivnyak's theorem, the statistical observation of a random point in a PPP $\Phi$ is the same as that of a typical (reference) point $x$ in the process $\Phi \cup x$.

In this work we assume high user mobility, implying that the probability of a ST being fully charged in the previous time slot and the probability of a ST being outside the guard zone in the current time slot are two independent probabilities. With the random access protocol, a ST that is allowed to be active has probability $p_{\mathrm{s}}$ to transmit in this time slot. Thus, the activation probability of a ST is given by

$$
p_{\mathrm{tr}}=p_{\mathrm{ch}} p_{\mathrm{gz}} p_{\mathrm{s}} .
$$

In each network realization, $p_{\text {tr }}$ represents the percentage of active secondary transmissions among all the secondary nodes.

\section{Performance Analysis}

In this section, we study the secondary throughput and the primary delay in the considered network model. An optimization problem is cast so as to find the optimal secondary access probability and the primary guard zone radius that maximizes the secondary throughput under primary delay constraints.

\section{A. Secondary Throughput}

In order to define the secondary throughput, we start from deriving the active probability and the successful transmission probability of a typical ST in the network. In this work, we assume bursty packet arrivals at the primary node. When the PT does not have a packet to transmit to its destination, it transmits an empty packet that does not contain valid information to assist the STs to harvest RF energy. ${ }^{1}$

Based on the one-slot charging assumption, we model the battery status of a ST by a two-state Discrete Time Markov Chain (DTMC) as depicted in Fig 1. The state $E$ denotes that the battery is empty and $F$ denotes a fully charged battery. This battery charging model can be generalized to multislot charging cases by considering more states for the battery status.

\footnotetext{
${ }^{1}$ This charging policy ensures that the STs will be provided with energy to harvest even if the PT has no packet to transmit. In the extended version of this work, we will also consider the case where the PT remains silent when it has no packet to transmit.
} 


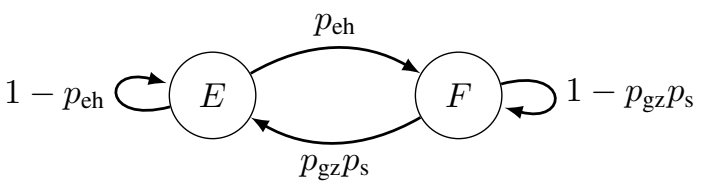

Fig. 1. The two-state Discrete Time Markov Chain that models the battery status of a secondary transmitter.

With the considered DTMC, it is easy to obtain the stationary probability that a secondary has a full battery. Then, the probability that a ST is charged, thus being able to transmit in the next time slot, is

$$
p_{\mathrm{ch}}=\frac{p_{\mathrm{eh}}}{p_{\mathrm{gz}} p_{\mathrm{s}}+p_{\mathrm{eh}}} .
$$

From basic geometry knowledge and the laws of cosine, we have

$$
\begin{gathered}
p_{\mathrm{eh}}=\frac{r_{\mathrm{eh}}^{2}}{R^{2}} \\
p_{\mathrm{gz}}=\left\{\begin{array}{cc}
r_{\mathrm{gz}} \leq R-d_{\mathrm{p}} \\
1-\frac{r_{\mathrm{gz}}^{2}}{R^{2}} . & r_{\mathrm{gz}}>R-d_{\mathrm{p}} \\
1-\frac{\phi r_{\mathrm{gz}}^{2}}{\pi R^{2}}-\frac{\varphi}{\pi}+\frac{d_{\mathrm{p}}}{\pi R} \sin \varphi,
\end{array}\right.
\end{gathered}
$$

where $\phi=\arccos \left(\frac{d_{\mathrm{p}}^{2}+r_{\mathrm{gz}}^{2}-R^{2}}{2 d_{\mathrm{p}} r_{\mathrm{gz}}}\right)$ and $\varphi=\arccos \left(\frac{R^{2}+d_{\mathrm{p}}^{2}-r_{\mathrm{gz}}^{2}}{2 R \cdot d_{\mathrm{p}}}\right)$.

We characterize the secondary throughput by the average number of successfully transmitted packets per time slot per unit area $\left(\right.$ packet $\left./ \mathrm{slot} / \mathrm{m}^{2}\right)$. Denote $T_{\mathrm{s}}$ the secondary throughput, we have

$$
T_{\mathrm{s}}=\lambda_{\mathrm{s}} p_{\mathrm{tr}} p_{\mathrm{suc}, \mathrm{s}}=\lambda_{\mathrm{s}} \frac{p_{\mathrm{eh}}}{p_{\mathrm{gz}} p_{\mathrm{s}}+p_{\mathrm{eh}}} p_{\mathrm{gz}} p_{\mathrm{s}} p_{\mathrm{suc}, \mathrm{s}},
$$

where $p_{\text {suc,s }}$ denotes the success probability of a typical secondary transmission. Here, we consider the success probability evaluation based on the received signal-to-interference-plusnoise ratio (SINR), i.e., a transmission is successful if the received SINR exceeds a prescribed threshold $\theta$. Conditioning on having a typical secondary pair $i$ with the receiver centered at the origin, we have the SINR given as

$$
\operatorname{SINR}_{i}=\frac{P_{2}\left|h_{i, i}\right|^{2} d_{s}^{-\alpha}}{\sum_{j \in \Phi_{\text {tr } \backslash\{i\}}} P_{2}\left|h_{j, i}\right|^{2} d_{j, i}^{-\alpha}+P_{1}\left|h_{0, i}\right|^{2} d_{0, i}^{-\alpha}+\sigma^{2}},
$$

where $\Phi_{\text {tr }}$ denote the set of active STs; $\left|h_{j, i}\right|^{2}$ denotes the small-scale power fading from the transmitter $j$ to the receiver $i$, which follows exponential distribution (Rayleigh fading) with unit mean value; $d_{j, i}$ denotes the distance between the transmitter $j$ to the receiver $i$. We assume a distance-dependent power law $d^{-\alpha}$ for the pathloss attenuation, where $\alpha>2$ denotes the pathloss exponent; $\sigma^{2}$ denotes the background noise power.

Due to the existence of the guard zone, the secondary interference field is not isotropic, i.e., the STs closer to the guard zone boundary will have higher success probability as a result of less received interference. In a two-tier hierarchical PPP network with guard zones around the primary PTs, some bounds and approximations on the interference distribution of a Poisson Hole Process (PHP) can be found in [18]. The exact secondary interference distribution in the considered network region with one single hole is out of the scope of this work. In this work, we only consider the case where $r_{\mathrm{gz}} \ll R$. Thus, a tight lower bound of $p_{\text {suc,s }}$ can be obtained by considering the distribution of the active interfering STs as a homogeneous PPP with intensity $\lambda_{s} p_{\mathrm{ch}} p_{\mathrm{s}}$. Hence, the success probability of the secondary transmission is given by

$$
\begin{aligned}
p_{\mathrm{suc}, \mathrm{s}} & =\mathbb{P}\left[\mathrm{SINR}_{i}>\theta\right] \\
& \stackrel{(a)}{=} \exp \left(-\theta d_{s}^{\alpha} I_{n}\right) \mathbb{E}_{d_{0, i}}\left[\frac{1}{1+\frac{\theta P_{1} d_{s}^{\alpha}}{P_{2} d_{0, i}^{\alpha}}}\right] \exp \left(-\frac{\theta \sigma^{2} d_{s}^{\alpha}}{P_{2}}\right) \\
& \stackrel{(b)}{\simeq} \mathcal{L}_{I_{n}}\left(d_{s}^{\alpha} I_{n}\right) \frac{\exp \left(-\frac{\theta \sigma^{2} d_{s}^{\alpha}}{P_{2}}\right)}{1+\frac{d_{s}^{2}}{\mathbb{E}\left[d_{0, i}\right]^{2}}\left(\theta \frac{P_{1}}{P_{2}}\right)^{\frac{2}{\alpha}}} \\
& \stackrel{(c)}{\approx} \exp \left(-\frac{\pi \lambda_{s} p_{\mathrm{ch}} p_{\mathrm{s}} d_{s}^{2} \theta^{\frac{2}{\alpha}}}{\operatorname{sinc}(2 / \alpha)}\right) \frac{\exp \left(-\frac{\theta \sigma^{2} d_{s}^{\alpha}}{P_{2}}\right)}{1+\frac{d_{s}^{2}}{\mathbb{E}\left[d_{0, i}\right]^{2}}\left(\theta \frac{P_{1}}{P_{2}}\right)^{\frac{2}{\alpha}}}
\end{aligned}
$$

where $I_{n}=\sum_{j \in \Phi_{\mathrm{tr}}}\left|h_{j, i}\right|^{2} d_{j, i}^{-\alpha}$ denotes the received secondary interference at the typical SR with normalized power, and $\mathcal{L}_{I_{n}}(s)=\mathbb{E}\left[e^{-s I_{n}}\right]$ denotes the Laplace transform of interference. Here, $(a)$ comes from the pdf of $\left|h_{i, i}\right|^{2}$, which follows exponential distribution; $(b)$ follows from the definition of Laplace transform of interference and the approximation used in [19]; $(c)$ follows from the probability generating functional (PGFL) of a PPP. Since the STs are randomly distributed in the network region, a random SR can be considered as randomly and uniformly distributed in $\mathcal{B}(0, R)$. The expectation of the distance between the PT and a random SR is thus $\mathbb{E}\left[d_{0, i}\right]=\frac{2 R}{3}$.

\section{B. Primary Delay}

The primary queue is modeled by a DTMC, as shown in Fig. 2, where $\lambda$ denotes the packet arrival rate and $\mu_{p}$ denotes the service rate. When assuming slotted transmission, the service rate of the primary node is equal to the success probability of the primary packet transmission in a time slot. Following similar definition of the SINR in (III-A), we have

$$
\mathrm{SINR}_{0}=\frac{P_{1}\left|h_{0,0}\right|^{2} d_{p}^{-\alpha}}{\sigma^{2}+\sum_{j \in \Phi_{a} \backslash \mathcal{B}\left(y_{0}, r_{\mathrm{gz}}\right)} P_{2}\left|h_{j, 0}\right|^{2} d_{j, 0}^{-\alpha}}
$$

where the index 0 denotes the primary node. For a given SINR target $\theta$, following similar steps as in the secondary success 
probability analysis, we have

$$
\begin{aligned}
\mu_{\mathrm{p}} & =\mathbb{P}\left[\operatorname{SINR}_{0}>\theta\right] \\
& =\exp \left(-\frac{\theta d_{p}^{\alpha} P_{2}}{P_{1}} I_{s}\right) \exp \left(-\frac{\theta \sigma^{2} d_{p}^{\alpha}}{P_{1}}\right) \\
& =\mathcal{L}_{I_{s}}\left(\frac{\theta d_{p}^{\alpha} P_{2}}{P_{1}}\right) \exp \left(-\frac{\theta \sigma^{2} d_{p}^{\alpha}}{P_{1}}\right) \\
& =\mathcal{L}_{I}^{1}\left(\frac{\theta d_{p}^{\alpha} P_{2}}{P_{1}}, \lambda_{s} p_{\text {tr }}, r_{\mathrm{gz}}\right) \exp \left(-\frac{\theta \sigma^{2} d_{p}^{\alpha}}{P_{1}}\right),
\end{aligned}
$$

where $I_{s}=\sum_{j \in \Phi_{\mathrm{tr}}} P_{2}\left|h_{j, 0}\right|^{2} d_{j, 0}^{-\alpha}$ denotes the secondary interference received at the $\mathrm{PR}, \mathcal{L}_{I}^{1}\left(s, \lambda_{a}, r\right)$ is defined as [20]

$$
\mathcal{L}_{I}^{1}\left(s, \lambda_{a}, r\right)=\exp \left(-2 \pi \lambda_{a} \int_{r}^{\infty} \frac{s v^{-\alpha}}{1+s v^{-\alpha}} v \mathrm{~d} v\right)
$$

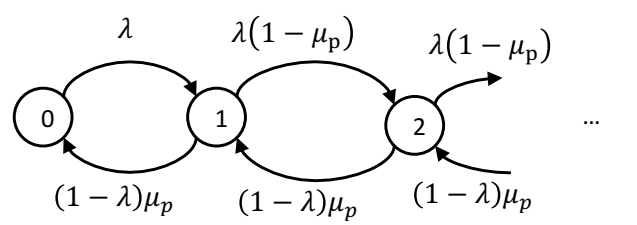

Fig. 2. The Discrete Time Markov Chain that models the evolution of the queue at the primary transmitter.

The primary queue is stable when $\lambda<\mu_{p}$ [21]. The distribution of the probability that the primary queue has $i$ packets, is given by

$$
\begin{aligned}
& \mathbb{P}[Q=0]=1-\frac{\lambda}{\mu_{p}} \\
& \mathbb{P}[Q=i]=\frac{\lambda^{i}\left(1-\mu_{p}\right)^{i-1}}{(1-\lambda)^{i} \mu_{p}^{i}} \mathbb{P}[Q=0] .
\end{aligned}
$$

Thus, we obtain the average queue size as follows

$$
\bar{Q}=\sum_{i=0}^{\infty} i \mathbb{P}[Q=i]=\frac{\lambda(1-\lambda)}{\mu_{p}-\lambda} .
$$

Denote $\bar{D}_{\mathrm{p}}$ the primary average delay, from Little's law [22], we have

$$
D_{p}=\frac{\bar{Q}}{\lambda}+\frac{1}{\mu_{\mathrm{p}}} .
$$

The intuition behind (5) and (13) is that the PT will be able to charge the STs within the EH zone, which increases the network throughput and therefore has the potential to improve the spectrum reuse. However, the active STs create interference to the primary transmission, which in turn increases the average primary queue size and delay.

In a cognitive shared access network with delay-sensitive primary user, we assume the following constraint for the average primary delay

$$
D_{\mathrm{p}} \leq D_{\max },
$$

where $D_{\max }$ is the maximum average delay that a primary user can tolerate.

\section{Secondary Throughput Optimization under Primary Delay} Constraints

We formulate the optimization problem in order to find the optimal secondary access probability $q_{s}$ and optimal primary guard zone radius $r_{\mathrm{gz}}$ that maximizes the secondary throughput with respect to primary delay constraints.

$$
\left(r_{\mathrm{gz}}^{*}, p_{s}^{*}\right)=\underset{\left(r_{\mathrm{gz}}, p_{s}\right)}{\arg \max } T_{s}
$$

subject to

$$
\begin{aligned}
& 0 \leq p_{s} \leq 1 \\
& 0 \leq r_{\mathrm{gz}} \leq R, \\
& D_{\mathrm{p}}\left(r_{\mathrm{gz}}, p_{s}\right) \leq D_{\max } .
\end{aligned}
$$

Since the secondary throughput is not a convex function, we resort to numerical methods to find the optimal guard zone radius and secondary access probability under primary delay constraints. The results obtained with different network parameter values will be presented in Section IV.

\section{NumericAl Results}

In this section, we evaluate the performance of the cognitive shared access network with energy harvesting. We consider a circular network region $\mathcal{C}$ with radius $R=300 \mathrm{~m}$. The PT is located at the center of $\mathcal{C}$, while the PR is located at distance $d_{p}=150 \mathrm{~m}$ from the PT. The STs are distributed according to a homogeneous PPP with intensity $\lambda_{\mathrm{s}}=0.001 / \mathrm{m}^{2}$. The SRs are located at isotropic directions around their associated STs with fixed secondary link distance $d_{s}=40 \mathrm{~m}$. The primary and secondary transmission powers are $P_{1}=10 \mathrm{~mW}$ and $P_{2}=0.1 \mathrm{~mW}$, respectively. The noise power is $\sigma^{2}=121$ $\mathrm{dBm}$. The SINR target for successful transmission is $\theta=5 \mathrm{~dB}$. The pathloss exponent is $\alpha=3.7$. The maximum primary average delay is $D_{\max }=4$ slots/packet.

\section{A. Secondary Throughput under Primary Delay Constraints}

We present in Fig. 3 and Fig. 4 the 3-D plot of the secondary throughput as a function of $p_{s}$ and $r_{\mathrm{gz}}$. The missing part in each plot corresponds to the regime where the primary delay constraint is not satisfied, i.e., $D_{p}>D_{\max }$. Fig. 3 and Fig. 4 are obtained with large EH zone $\left(r_{\text {eh }}=100 \mathrm{~m}\right)$ and with small EH zone $\left(r_{\mathrm{eh}}=50 \mathrm{~m}\right)$, respectively. In both figures, the point that gives the largest $T_{s}$ corresponds to the optimal $p_{s}$ and $r_{\mathrm{gz}}$ as the solution to the optimization problem defined in (15).

By comparing these two figures, we see that when $r_{\mathrm{eh}}=100$ $\mathrm{m}$, the optimal access probability $p_{s}$ which gives the highest $T_{s}$ is less than 1 . However, when $r_{\text {eh }}=50 \mathrm{~m}$, the optimal access probability $p_{s}$ is 1 , since $T_{s}$ in Fig. 4 always increases with $p_{s}$. This comes from the fact that when the EH zone is small, the density of charged STs is small, which falls in the regime where increasing the density of active secondary nodes always increases the throughput. On the other hand, when the $\mathrm{EH}$ zone is large, due to the relatively high density of charged STs, maximizing the secondary throughput requires that each charged node accesses the spectrum with a certain probability to control the interference level. 


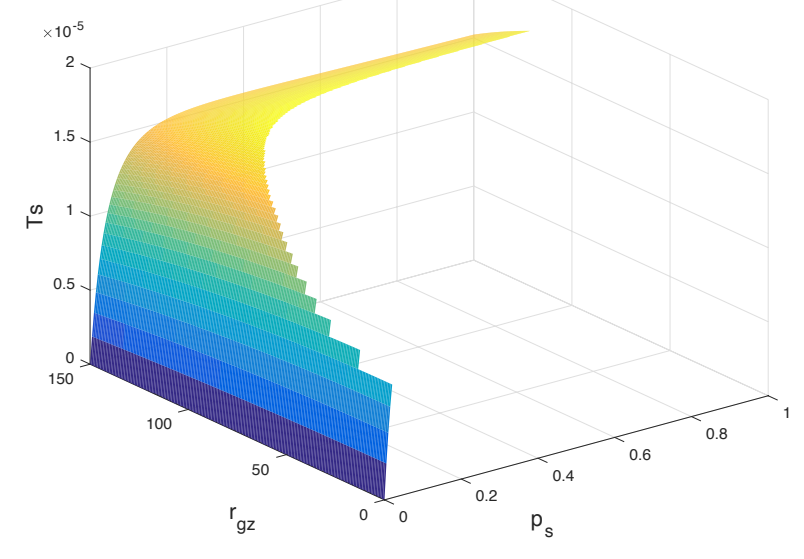

Fig. 3. Secondary throughput vs. $\left(p_{s}, r_{\mathrm{gz}}\right) . \lambda=0.5 . r_{\mathrm{eh}}=100 \mathrm{~m}$.

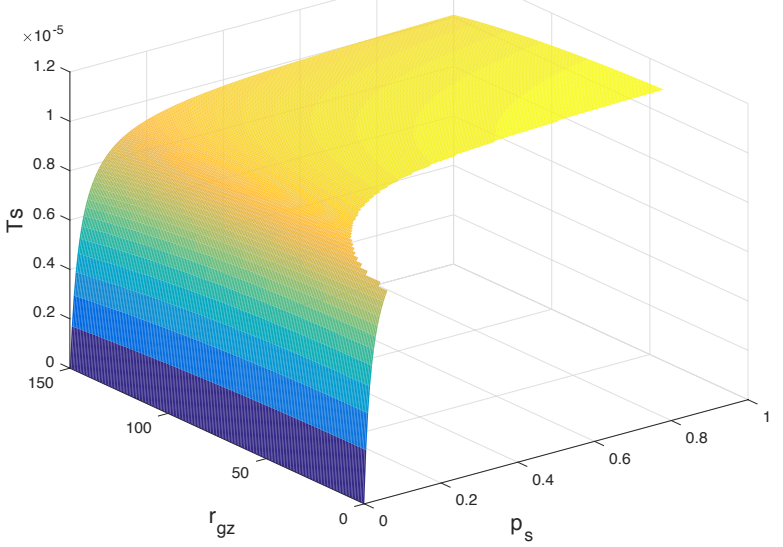

Fig. 4. Secondary throughput vs. $\left(p_{s}, r_{\mathrm{gz}}\right) . \lambda=0.5 . r_{\mathrm{eh}}=50 \mathrm{~m}$.

\section{B. Primary Average Delay}

We present in Fig. 5(a) and Fig. 5(b) the primary average delay as a function of the secondary access probability $p_{s}$ for different values of $r_{\mathrm{gz}}$. The $\mathrm{EH}$ zone radius is chosen as $r_{\mathrm{eh}}=80 \mathrm{~m}$. The two figures represent the cases with low and high packet arrival rates, respectively. From these figures, it is obvious that when the guard zone size is sufficiently large, the primary delay is not very sensitive to the density of active secondary nodes. From the primary delay perspective, increasing the guard zone radius is more efficient in terms of interference mitigation. However, combined with Fig. 3 and Fig. 4, we see that increasing $r_{\mathrm{gz}}$ lowers the maximum achievable secondary throughput. Hence, there is a tradeoff between the maximum secondary throughput and primary delay that must be taken into account when choosing the optimal value of $r_{\mathrm{gz}}$.

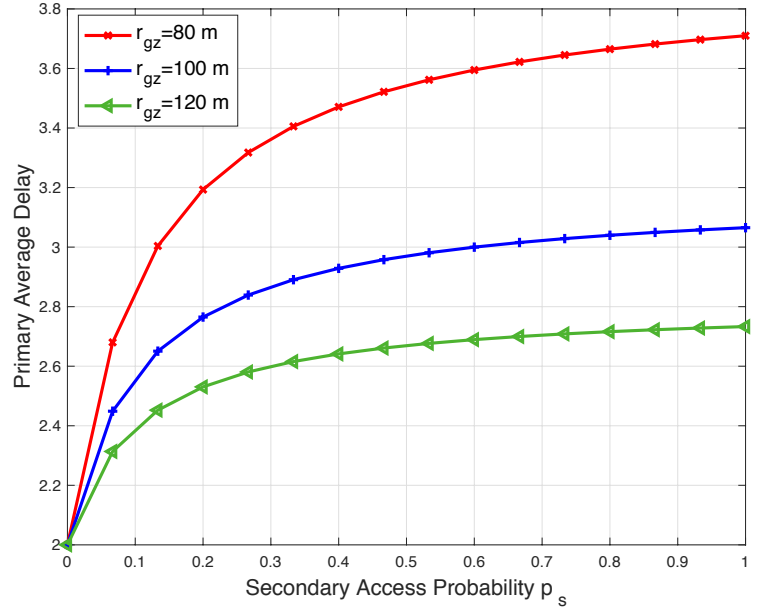

(a) With low packet arrival rate, i.e., $\lambda=0.3$.

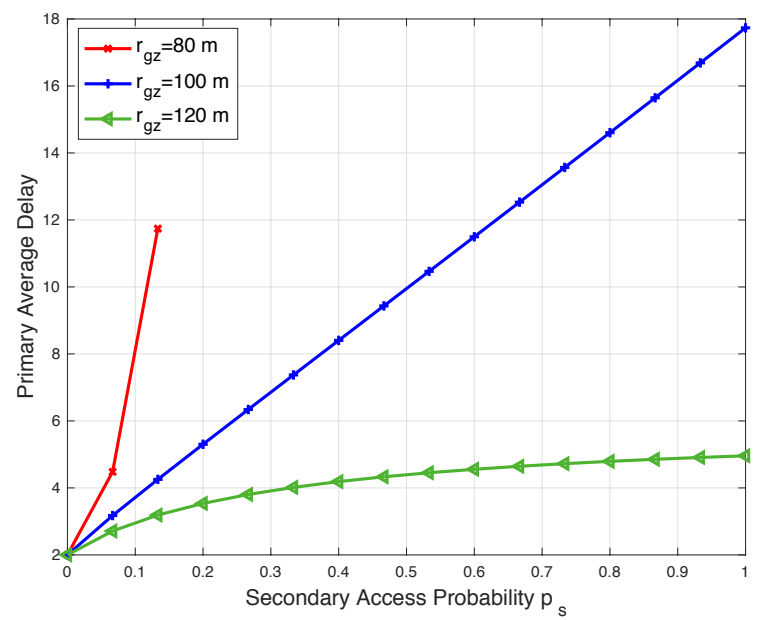

(b) With high packet arrival rate, i.e., $\lambda=0.7$.

Fig. 5. Primary delay vs $p_{s}$ for different values of guard zone radius $r_{\mathrm{gz}}$. EH zone radius $r_{\mathrm{eh}}=80 \mathrm{~m}$.

TABLE I

OPTIMAL $p_{s}$ AND $r_{\mathrm{GZ}}$ IN DIFFERENT CASES

\begin{tabular}{|c|c|c|c|c|}
\hline$r_{\mathrm{eh}}(\mathrm{m})$ & $\lambda$ & $q_{s}^{*}$ & $r_{\mathrm{gz}}^{*}(\mathrm{~m})$ & $T_{s}^{*}\left(\times 10^{-5}\right)$ \\
\hline \multirow{3}{*}{100} & 0.3 & 0.4 & 89 & 1.87 \\
\cline { 2 - 5 } & 0.5 & 0.313 & 106 & 1.7868 \\
\cline { 2 - 5 } & 0.7 & 0.222 & 135 & 1.614 \\
\hline \multirow{3}{*}{50} & 0.3 & 1 & 0 & 1.1053 \\
\cline { 2 - 5 } & 0.5 & 1 & 44 & 1.0987 \\
\cline { 2 - 5 } & 0.7 & 1 & 74.2 & 1.0858 \\
\hline
\end{tabular}

\section{Optimal Values of $p_{s}$ and $r_{g z}$}

In Table I, we provide the numerical results of the optimal $p_{s}$ and $r_{\mathrm{gz}}$ that maximize the secondary throughput under primary delay constraints. We present the numerical results for different $\mathrm{EH}$ size $r_{\mathrm{eh}}$ and different values of the packet arrival rate $\lambda$, in order to show the impact of these network 
parameters on the optimal solution to the problem defined in Section III-C.

First, we see that smaller EH zone radius leads to higher optimal access probability $q_{s}$ and smaller cellular guard zone size $r_{\mathrm{gz}}$. This result is as expected because larger $\mathrm{EH}$ zone leads to higher density of secondary devices that can be charged. When the density $p_{\text {ch }}$ reaches a certain level, the optimal access probability $q_{s}$ will decrease when $p_{\text {ch }}$ increases. Second, we notice that the maximum secondary throughput $T_{s}^{*}$ is higher in the case with larger $\mathrm{EH}$ zone, despite that the optimal guard zone radius $r_{\mathrm{gz}}^{*}$ in this case is also larger.

\section{Conclusions}

In this paper, we investigated a cognitive shared access network with energy harvesting-based opportunistic secondary nodes. Assuming bursty traffic at the primary node, we studied the throughput and delay performance in such network, which depend on the energy harvesting zone and the secondary access protocol. We casted an optimization problem that aims at maximizing the secondary throughput with primary delay constraints. Through numerical results, we showed some interesting remarks about the impact of different protocol parameters on the optimized network performance.

\section{REFERENCES}

[1] S. Park, H. Kim, and D. Hong, "Cognitive radio networks with energy harvesting," IEEE Transactions on Wireless Communications, vol. 12, no. 3, pp. 1386-1397, Mar. 2013.

[2] S. Park and D. Hong, "Optimal spectrum access for energy harvesting cognitive radio networks," IEEE Transactions on Wireless Communications, vol. 12, no. 12, pp. 6166-6179, Dec. 2013.

[3] D. T. Hoang, D. Niyato, P. Wang, and D. I. Kim, "Opportunistic channel access and RF energy harvesting in cognitive radio networks," IEEE Journal on Selected Areas in Communications, vol. 32, no. 11, pp. 20392052, Nov. 2014.

[4] N. Pappas, J. Jeon, A. Ephremides, and A. Traganitis, "Optimal utilization of a cognitive shared channel with a rechargeable primary source node," Journal of Communications and Networks, vol. 14, no. 2, pp. 162-168, Apr. 2012.

[5] H. ElSawy, E. Hossain, and M. Haenggi, "Stochastic geometry for modeling, analysis, and design of multi-tier and cognitive cellular wireless networks: A survey," IEEE Communications Surveys Tutorials, vol. 15, no. 3, pp. 996-1019, Third 2013.

[6] K. Huang, "Spatial throughput of mobile ad hoc networks powered by energy harvesting," IEEE Transactions on Information Theory, vol. 59 no. 11, pp. 7597-7612, Nov. 2013

[7] Y. Yao, C. Yin, X. Song, and N. C. Beaulieu, "Increasing throughput in energy-based opportunistic spectrum access energy harvesting cognitive radio networks," Journal of Communications and Networks, vol. 18, no. 3, pp. 340-350, Jun. 2016.

[8] S. Lee, R. Zhang, and K. Huang, "Opportunistic wireless energy harvesting in cognitive radio networks," IEEE Transactions on Wireless Communications, vol. 12, no. 9, pp. 4788-4799, Sep. 2013.

[9] H. H. Yang, J. Lee, and T. Q. S. Quek, "Heterogeneous cellular network with energy harvesting-based D2D communication," IEEE Transactions on Wireless Communications, vol. 15, no. 2, pp. 1406-1419, Feb. 2016.

[10] A. H. Sakr and E. Hossain, "Cognitive and energy harvesting-based D2D communication in cellular networks: Stochastic geometry modeling and analysis," IEEE Transactions on Communications, vol. 63, no. 5, pp. 1867-1880, May 2015.

[11] N. Pappas, M. Kountouris, J. Jeon, A. Ephremides, and A. Traganitis, "Network-level cooperation in energy harvesting wireless networks," in IEEE Global Conference on Signal and Information Processing, Dec 2013, pp. 383-386.

[12] J. Jeon and A. Ephremides, "On the stability of random multiple access with stochastic energy harvesting," IEEE Journal on Selected Areas in Communications, vol. 33, no. 3, pp. 571-584, March 2015.
[13] N. Pappas, M. Kountouris, J. Jeon, A. Ephremides, and A. Traganitis, "Effect of energy harvesting on stable throughput in cooperative relay systems," Journal of Communications and Networks, vol. 18, no. 2, pp. 261-269, April 2016.

[14] Z. Chen, N. Pappas, M. Kountouris, and V. Angelakis, "Throughput analysis of smart objects with delay constraints," in IEEE 17th International Symposium on A World of Wireless, Mobile and Multimedia Networks (WoWMoM), June 2016, pp. 1-6.

[15] — "On the performance of delay aware shared access with priorities." [Online]. Available: http://arxiv.org/abs/1603.08885

[16] A. Hasan and J. G. Andrews, "The guard zone in wireless ad hoc networks," IEEE Transactions on Wireless Communications, vol. 6, no. 3, pp. 897-906, Mar. 2007.

[17] F. Baccelli, B. Blaszczyszyn, and P. Muhlethaler, "Stochastic analysis of spatial and opportunistic aloha," IEEE Journal on Selected Areas in Communications, vol. 27, no. 7, pp. 1105-1119, Sep. 2009.

[18] Z. Yazdanshenasan, H. S. Dhillon, M. Afshang, and P. H. J. Chong, "Poisson hole process: Theory and applications to wireless networks," IEEE Transactions on Wireless Communications, vol. 15, no. 11, pp. 7531-7546, Nov. 2016.

[19] N. Lee, X. Lin, J. G. Andrews, and R. W. Heath, "Power control for D2D underlaid cellular networks: Modeling, algorithms, and analysis," IEEE Journal on Selected Areas in Communications, vol. 33, no. 1, pp. $1-13$, Jan. 2015.

[20] Z. Chen and M. Kountouris, "Guard zone based D2D underlaid cellular networks with two-tier dependence," in IEEE International Conference on Communication Workshop (ICCW), June 2015, pp. 222-227.

[21] R. Loynes, "The stability of a queue with non-independent inter-arrival and service times," Proc. Camb. Philos. Soc, vol. 58, no. 3, pp. 497-520, 1962.

[22] D. Bertsekas and R. Gallager, Data networks (2nd ed.). Upper Saddle River, NJ, USA: Prentice-Hall, Inc., 1992. 